First publ. in: Physics Letters A 130 (1988), 1-3, pp. 401-404

\title{
INVESTIGATION OF LASER DESORPTION OF THIN DIELECTRIC FILMS USING OPTICALLY EXCITED SURFACE PLASMONS
}

\author{
S. PUDERBACH. S. HERMINGHAUS and P. LEIDERER \\ Institut fïr Physik, Jishannes (iutenberg-Universität. Mainz, FRG
}

Received 29 February 1988; accepted for publication 20 May 1988

Communicated by D. Bloch

Optically excited surfacc plasmons have been used as a probe for pulsed laser desorption of organic compounds from a metal surface. Changes in the adsorbed film thickness icss than $1 \AA$ are easily detected on a nanosecond time scale. Furthermore, a transient laser-induced increase of the dielectric constant of the adsorbate is observed.

Fast surface processes induced by pulsed laser irradiation have recently become a field of great interest. However, most of the techniques of surface analy'sis which are presently available do not provide the temporal resolution required to investigate those phenomena on a nanosecond scale. New methods in surface analysis as well as improvements in the time resolution of well-established techniques are thus desirable. In the experiments reported here optically excited surface plasmons (SP) have been used as a probe for pulsed laser desorption of organic compounds from a metal surface. By this method a change in layer thickness less than one ångström can easily be detected on a time scale which is in our case limited only by the electronics.

As is well known, SP can be excited optically by the so-called attenuated-total-reflectance (ATR) method [1] (see inset in fig. 1): a glass surface is illuminated with monochromatic light from the glass half space at an angle $\theta$ large enough for total reflection to occur. The evanescent light wave is coupled to a metal surface next to the glass. When the angle of incidence is varied, resonant coupling of the evanescent light field to the surface plasma mode of the metal takes place at a certain angle $\theta_{0}$, resulting in a substantial decrease of the reflected intensity due to SP excitation. This resonance effect is extremely sensitive to changes in the characteristics of the metal surface, such as roughness or coverage with a thin film [2,3] (see fig. 1). In particular, a dielectric film

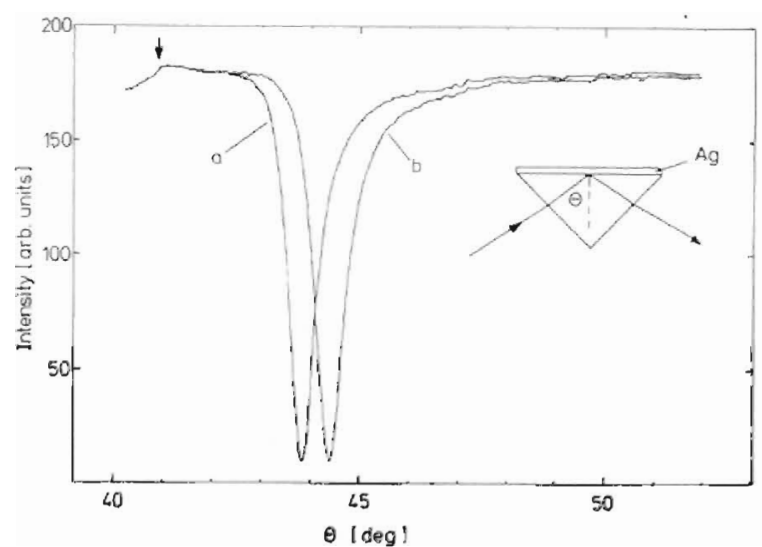

Fig. 1. The surface piasma kesonance observed on a silver surface covered with a dielectric film at $77 \mathrm{~K}$ (in this case water from the vacuum system), shown for two different film thicknesses: (a) $10 \AA$, (b) $70 \AA$. The retiected intensity is plotted versus the angle. of incidence. The arrow maris the internal angle of total reflection. A sketch of the samplc geometry is shown in the inset: The silver film covers the base of a $\mathrm{BK} 7$ glass prism, and the surface plasmons are excited at the silver-vacuum interface.

with thickness $d$ gives rise to a shift of the resonance angle $\theta_{0}$ by an amount $\Delta \theta_{0}$, which to first order is given by [4]

$\Delta \theta_{0}=C_{0} d(\epsilon-1)$.

Here $\epsilon$ is the dielectric constant of the film, and thc constant $C_{0}$ is of the order of $10^{-2} \mathrm{deg} / \AA$.

We have used a setup first proposed by Kretschmann [5], where the metal (in our case silver) forms 
a film $(d=500 \AA)$ on the glass surface. The light of a He-Ne laser $(\lambda=632.8 \mathrm{~nm})$ excites the SP at the metal-vacuum interface by the evanescent light field which penetrates the metal. The silver film was evaporated onto a prism base, which served as the glass substrate. The prism was thermally coupled to a liquid nitrogen reservoir $(T=77 \mathrm{~K})$, so that thin layers of dielectric material could be frozen onto the silver surface from the vapor phase. Our measurements were carried out for physisorbed films of 2 propanol $\left(\mathrm{C}_{3} \mathrm{H}_{7} \mathrm{OH}\right)$ and tetrafluoromethane $\left(\mathrm{CF}_{4}\right)$ typically $100 \AA$ thick. An excimer laser $(\mathrm{KrF} ; 248$ $n m$ ) was used for the desorption of the films.

In order to study the time dependence of the SP resonance characteristics during the desorption process, one needs a sequence of resonance curves, such as in fig. 1 , on a nanosecond time scale. Since it is difficult to scan the angle $\theta$ so rapidly, we have measured transients of the reflected intensity at different fixed angles, and have reconstructed the time dcpendent SP resonance curve from the set of transients. In this way the resonance angle as well as the width and the depth of the resonance can be obtained as time-dependent quantities, from which properties like the complex dielectric constant and the thickness of the adsorbate can be inferred. Here we restrict our considerations to the shift of the resonance angle.

2-propanol. A film of $\mathrm{C}_{3} \mathrm{H}_{7} \mathrm{OH}$ was evaporated onto the silver surface at $77 \mathrm{~K}$ in high vacuum and was then partially desorbed by the excimer laser pulse at a fluence of $12 \mathrm{~mJ} / \mathrm{cm}^{2}$. The laser pulse intensity and the reflectivity of the silver surface were eached measured with a high speed PIN-diode and recorded on a storage oscilloscope. As an example, fig. 2 shows a typical trace taken at an angle $\theta<\theta_{0}$, near the inflection point of the leading edge of the SP resonance curve. Under this condition one would expect a decrease in intensity due to the SP shift $\Delta \theta_{0}$ corresponding to the reduction of film thickness by the desorption process (see eq. (1)). For times larger than 100 ns a decrease in intensity is observed indeed, but first a pronounced peak appears. This feature indicates that apart from desorption an additional process is induced by the laser pulse, connected with a temporary increase in $\theta_{0}$ and hence in the quantity $d(\epsilon-1)$.

The shift of the resonance angle as evaluated from

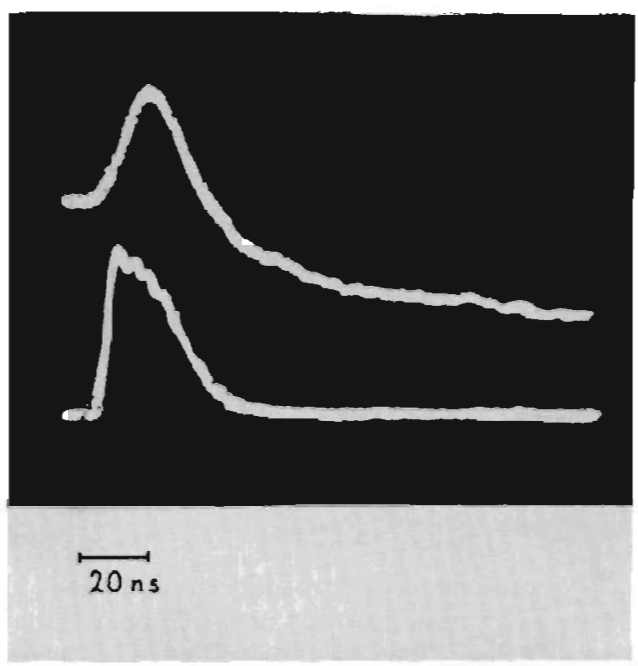

Fig. 2. Typical storage oscilloscope traces, taken at a fixed angle of incidence $\theta<\theta_{0}$. Upper curve: reflectivity transient; lower curve: laser intensity.

a set of transients like the one in fig. 2 is plotted in fig. 3a. According to eq. (1) the thickness $d$ desorbed by one pulse can be determined numerically from the asymptotic value of $\Delta \theta_{0}$, which in the present case yields $d=6.1 \pm 0.5 \AA$. (We have used here a value of 2.23 for the dielectric constant of 2-propanol, obtained by extrapolation from the room temperature value [6]. The total thickness of the layer as determined from the resonance angle $\theta_{0}$ was $113 \AA$. .)

We interpret the shift towards larger resonance angles during the first tens of nanoseconds as being due to highly polarizable states of the adsorbed molecules, excited by the pulse laser ultraviolet irradiation. This interpretation is substantiated by the data obtained for $\mathrm{CF}_{4}$, as discussed below.

Tetrafluoromethane. Fig. 3b shows the time dependent resonance shift for films of $\mathrm{CF}_{4}$. Obviously, this material is not noticeably desorbed by the laser pulses applied here, because for long times (even longer than the $5 \mu$ s displayed in fig. 3) $\Delta \theta_{0}$ asymptotically approaches zero within our accuracy of $\pm 5 \times 10^{-3} \mathrm{deg}$. This implics that the ablation per pulse has to be less than $0.5 \AA$. By making use of the cumulative effect of a sequence of pulses this upper limit could be further reduced to $\Delta d \leqslant 0.01 \AA$ per pulse.

The transient shift towards larger $\theta_{0}$, on the other 

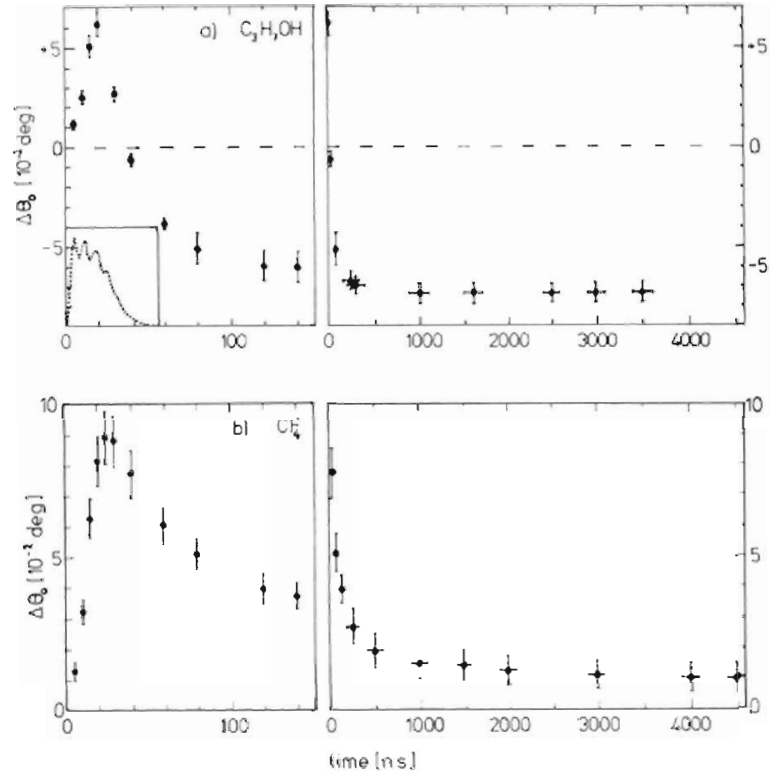

Fig. 3. Left: shift of the resonance angle, $\Delta \theta_{0}$, as reconstructed from sets of reflectivity transients like the one in fig. 2 for (a) 2 propanol. (b) tetrafluoromethane. The dotted line in the inset of (a) represents the intensity of the excimer laser pulse on the samc time scale. The right hand sides show $\Delta \theta_{0}$ for a larger time interval, measured with a second storage oscilloscope. The error bars are due to fluctuations of the laser pulse energy. For $\mathrm{CF}_{4}$ the value of $\Delta \theta_{0}$, which is still $1.0 \times 10^{-2}$ deg at $5 \mu \mathrm{s}$, asymptotically approaches zero at longer times.

hand, occurs for $\mathrm{CF}_{4}$ in a similar way as for propanol. We thus conclude that also here highly polarizable states are excited by the excimer laser pulse. Since an ablation signal is not superimposed in this case, a quantitative discussion of the observed decay appears to be easier, and we will therefore concentrate in the following on the data for $\mathrm{CF}_{4}$.

In order to analyze the time dependence of the SP resonance angle shift, we have replotted the data on a semi-logarithmic scale in fig. 4. Clearly an exponential decay characterized by a single relaxation time, which would correspond to a straight line in this plot, does not fit the data points. However, a very good fit is achieved by the solid line, which represents a simple model: The UV laser pulse dissociates the molecules into separate radicals with high polarizability, which recombine after a random walk in the film. Under the assumption that the number density $N$ of the radicals is much smaller than the total number density of the $\mathrm{CF}_{4}$ molecules we expect

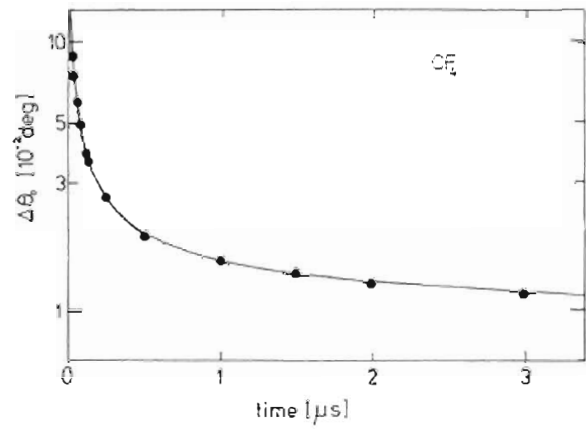

Fig. 4. Decay of the resonance shift for $\mathrm{CF}_{4}$ on a semi-logarithmic scale. The solid line represents a model based on the recombination of photodissociation products as described in the text.

a change in the dielectric constant according to the Clausius-Mosotti equation

$\Delta(\epsilon-1) \propto N$,

and therefore from eq. (1)

$\Delta \theta_{0} \propto N$,

since the film thickness $d$ is essentially constant in this case. The random walk model gives the following expression for the number density of radicals [7]:

$\log \left(N / N_{0}\right)=-C_{1} \int_{0}^{1} P\left(t^{\prime}\right) \mathrm{d} t^{\prime}$,

with

$P(t)=(8 \pi D t)^{-3 / 2} \exp \left(-\delta^{2} / 8 D t\right)$,

when fission into two radicals is assumed. $D$ is here the diffusion constant, $\delta$ the mean distance of the fission products at the end of the laser pulse, and the constant $C_{1}$ characterizes the recombination process itself. The fit yields

$\delta=(313 \mathrm{~ns})^{1 / 2} D^{1 / 2}$

and

$D=(6.6 \mathrm{~ms})^{-1 / 3} C_{1}^{2 / 3}$.

(For further analysis, information about the recombination constant $C_{1}$ will be required.)

In the case of propanol the above model gives much less satisfactory agreement with the data. We ascribe this fact to the superimposed desorption process (for early times) and no the larger variety of possible fis- 
sion products and the more complicated chemistry of this system.

In conclusion, we have shown that SP resonance can be used as a fast and sensitive tool for studying the processes involved in laser desorption of dielectric film from a metal surface. Both the ablation and the excitation of the film material could be observed. In the case of tetrafluoromethane, the data can be described with a simple radical diffusion model. For further studies considerable improvement in accuracy seems to be possible. More details of this work will be published elsewhere.

We would like to thank Professor W. Knoll and the groups of Professor K. Binder and Professor P. Guetlich for stimulating discussions. This work was supported by the Materialwissenschaftl. Forschungs- zentrum at the Johannes Gutenberg University of Mainz and by the Deutsche Forschungsgemeinschaft, SFB 252.

\section{References}

[1] A. Otto, in: Advanced optical properties of solids. New dcvelopments, ed. B.O. Seraphin (North-Holland, Amsterdam, 1976) p. 678.

[2] A. Humbert and J. Hanus, Surf. Sci. 129 (1983) 265.

[3] T. Kloos, Z. Phys. 208 (1968) 77.

[4] I. Pockrand, Surf. Sci. 72 (1978) 577.

[5] E. Kretschmann and H. Raether, Z. Naturforsch. 23a (1968) 2135.

[6] R.C. Wcast, cd., Handbook of chemistry and physics, $67 \mathrm{hh}$ Ed. (1986/1987) p. C-447.

[7] L.D. Landau and E.M. Lifshitz, Fluid mechanics (Pergamon, Oxford, 1959). 